sustaining arguments, and is thus a first step towards the validation of the calibration as a whole.

\section{Pion capture reveals alpha clusters}

from our Nuclear Theory Correspondent

WHEN a negative pion comes to rest it is captured by a nucleus and gives it all its rest energy. Since energy and momentum must be conserved in the capture process the pion cannot be captured by a single nucleon but must interact with several nucleons at once, so a detailed study of the particles emitted provides information on nucleon correlations in the nucleus.

One of the most likely forms of nucleon correlation is their tendency, especially in light nuclei, to form $\alpha$ clusters, and a recent experiment by Lewis, Ullrich, Engelhardt and Boschitz (Phys. Lett., 47B, 339; 1973) on the capture of negative pions by ${ }^{16} \mathrm{O}$ provides new evidence of the presence of these clusters in nuclei.

They looked at the $\gamma$-ray spectrum emitted by a ${ }^{16} \mathrm{O}$ target irradiated with slow pions and found a strong peak due to the $4.44 \mathrm{MeV} \gamma$-ray from the decay of the first $2^{+}$excited state of ${ }^{12} \mathrm{C}$. It is thus natural to suppose that the pion capture has broken the ${ }^{16} \mathrm{O}$ into an $\alpha$ particle and an excited ${ }^{12} \mathrm{C}$ nucleus, and indeed it is known that this mode of breaking up is about six times more likely than the corresponding process leaving ${ }^{12} \mathrm{C}$ in its ground state.

Since the $\alpha$ particle has a momentum distribution relative to the ${ }^{12} \mathrm{C}$ in the original oxygen nucleus, the recoiling carbon nuclei have the same distribution, superposed on the recoil momentum determined by the rest mass of the pion. This momentum distribution produces a Doppler broadening of the shape of the $\gamma$-ray line, and measurement of this broadening gives the momentum distribution of the $\alpha$ particles.

This momentum distribution can also be calculated from a simple harmonic oscillator model, thus providing a check on the interpretation of the data. In such a model, the momentum distribution of a particle moving with angular momentum $L$ with respect to the centreof-mass of the harmonic oscillator potential has the form $P(K) \propto K^{2^{L}}$ $\exp \left(-K^{2} / 2 Q_{L}{ }^{2}\right)$, and fits to the data given $Q_{2}=75 \pm 5 \mathrm{MeV} / c$. This may be compared with analyses of the ${ }^{16} \mathrm{O}(\mathrm{p}, \mathrm{p} \alpha)$ knockout reaction that give $Q_{0} \approx 75$ $\mathrm{MeV} / c$. The values of $Q_{0}$ and $Q_{2}$ are expected to be similar, so this is an indication of the consistency of the two analyses.

This experiment essentially gives the distribution of the sum of the momenta of the four nucleons emitted when pions are captured by ${ }^{16} \mathrm{O}$. These four nucleons are most probably combined to form an $\alpha$ particle because the alternative interpretation of absorption on a deuteron followed by evaporation of two nucleons would give the characteristic $\gamma$-rays from the decay of ${ }^{12} \mathrm{~B}$, and these are not observed. Other possible origins of the observed $\gamma$-rays were excluded by repeating the experiment with different targets.

It is thus very reasonable to interpret these observations by the $\alpha$ particle absorption model and this gives further support to the idea that $\alpha$ clusters are present in nuclei. Similar results have been obtained for other nuclei besides oxygen.

\section{Population energetics of kangaroo rats}

from our Animal Ecology Correspondent

IT has been suggested that because terrestrial herbivores have low efficiencies of consumption of net primary production (NPP), their populations generally are not limited by food (Slobodkin et al., Am. Nat., 101, 109; 1966; Wiegert and Owen, J. theor. Biol., 30, 69 ; 1971). Most species of small mammals from the temperate biomes consume between $0.1 \%$ and $1.0 \%$ of the NPP annually. When this amount is expressed in terms of available productivity (AP)-that is, ignoring inedible parts of the plant-the values range from $0.1 \%$ to $4.0 \%$, with a few exceptions (Chew and Chew, Ecol. Monogr., 40, 1; 1970; Wiegert and Evans, in Secondary Productivity in Terrestrial Ecosystems (edit. by K. Petrusewicz) 499, Polish Academy of Science, Warsaw, 1967; Grodzinski et al., Acta Theriol., 11, 419; 1966). Soholt has now published some interesting data on the impact of a population of the kangaroo rat (Dipolomys merriami) on the Mojave desert and shows that the rats take $6.9 \%$ of the NPP and $10.7 \%$ of the AP annually (Ecol. Monogr., $43,357 ; 1973)$. The study offers some thoughts on why population densities of desert rodents are as high as they are.

Soholt estimated population density by recapture trapping and the Lincoln Index. During the year of study the density averaged 16.2 rats $\mathrm{ha}^{-1}$, a high figure for a species living in conditions where the NPP is just 1,400 megacal $\mathrm{ha}^{-1} \mathrm{yr}^{-1}$. (In temperate zones the NPP is between 20,000 and 30,000 megacal $\mathrm{ha}^{-1} \mathrm{yr}^{-1}$.) Soholt measured resting energy expenditure, the energy cost of activity, the energy expense of growth, assimilation efficiency and calculated the total energy flow $(E)$ through the population to be 85.5 megacal ha-1 $\mathrm{yr}^{-1}$.
From his studies on growth and reproduction he deduced that the secondary productivity $(P)$ of the population was 0.7 megacal ha-1 $\mathrm{yr}^{-1}$, or $0.8 \%$ of the total energy flow. This is somewhat lower than the $P / E$ ratio for other rodents which fluctuates between 1.2 and $3.0 \%$. This suggests that kangaroo rats are not adapted for utilisation of as high a proportion of their total energy flow for secondary productivity as are non-desert species. Perhaps this adaptation is prudential in an environment where the amount of food production is unpredictable.

Although several species of plants were available to the rodents, they showed a strong preference for the annual storksbill Erodium cicutarium. Seeds formed the major item in the diet, but leaves were consumed during the winter. Laboratory studies showed the assimilation efficiency $(A E)$ to be 0.87. Assuming this, the amount of food that had to be consumed such that $E / A E$ equaled 85.5 megacal $\mathrm{ha}^{-1} \mathrm{yr}^{-1}$ was calculated to be 97.8 megacal $\mathrm{ha}^{-1}$ $\mathrm{yr}^{-1}$. A little more than 1.0 megacal was supplied by arthropods; most of the rest by Erodium.

Further field studies revealed that, in order to obtain this calorific input chiefly from one species of plant, the rats consumed, incredibly, more than $95 \%$ of the Erodium seed production and more than $90 \%$ of the total Erodium production. The population thus exerted a massive effect on the energy turnover of this one species, although at $6.9 \%$ of the total NPP it had little effect on the vegetation as a whole. Its impact on the survival chances of this species population would be immense if the remaining $5 \%$ of the seeds are insufficient to support as great a plant population in the succeeding year. It is a pity that the study was not continued to ascertain what were the population levels of rat and storksbill during the following year. Populations of desert annuals are limited by rainfall so that desert rodents are unlikely to exert an important controlling effect. If rainfall was not limiting, the rodents would likely consume Erodium until it became scarce to find and in that way actually limit its population.

Soholt's study shows that this population of Dipodomys lived dangerously on the brink of overexploiting its food resources. Wiegert and Owen suggested that low consumption of plants avoided long term oscillations of the plant populations. In deserts, where extremes of environmental factors make oscillations unavoidable, the evolution of such a damper would prove ineffective. Thus there is no disadvantage to a population of rodents consuming more than $95 \%$ of its major food source. 